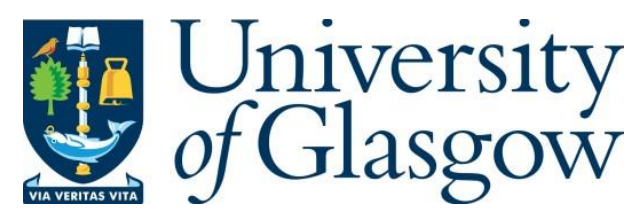

Menditto, R., Sickinger, H., Weides, M., Kohlstedt, H., Žonda, M., Novotný, T., Koelle, D., Kleiner, R. and Goldobin, E. (2016) Phase retrapping in a $\varphi$ Josephson junction: onset of the butterfly effect. Physical Review B, 93, 174506.

There may be differences between this version and the published version. You are advised to consult the publisher's version if you wish to cite from it.

http://eprints.gla.ac.uk/155844/

Deposited on: 25 May 2018

Enlighten - Research publications by members of the University of Glasgow http://eprints.gla.ac.uk 


\title{
Phase retrapping in a $\varphi$ Josephson junction: onset of the butterfly effect
}

\author{
R. Menditto, ${ }^{1}$ H. Sickinger ${ }^{1}$ M. Weides,${ }^{2}$ H. Kohlstedt,${ }^{3}$ M. \\ Žonda, ${ }^{4}$ T. Novotný, ${ }^{4}$ D. Koelle, ${ }^{1}$ R. Kleiner, ${ }^{1}$ and E. Goldobin ${ }^{1}$ \\ ${ }^{1}$ Physikalisches Institut and Center for Collective Quantum Phenomena in LISA ${ }^{+}$, \\ Universität Tübingen, Auf der Morgenstelle 14, D-72076 Tübingen, Germany \\ ${ }^{2}$ Peter Grünberg Institute and JARA-Fundamentals of Future Information Technology, \\ Forschungszentrum Jülich GmbH, 52425 Jülich, Germany* \\ ${ }^{3}$ Nanoelektronik, Technische Fakultät, Christian-Albrechts-Universität zu Kiel, D-24143 Kiel, Germany \\ ${ }^{4}$ Department of Condensed Matter Physics, Faculty of Mathematics and Physics, \\ Charles University in Prague, Ke Karlovu 5, 12116 Praha 2, Czech Republic
}

(Dated: April 21, 2016)

\begin{abstract}
We investigate experimentally the retrapping of the phase in a $\varphi$ Josephson junction upon return of the junction to the zero-voltage state. Since the Josephson energy profile $U_{0}(\psi)$ in $\varphi$ JJ is a $2 \pi$ periodic double well potential with minima at $\psi= \pm \varphi \bmod 2 \pi$, the question is at which of the two minima $-\varphi$ or $+\varphi$ the phase will be trapped upon return from a finite voltage state during quasistatic decrease of the bias current (tilt of the potential). By measuring the relative population of two peaks in escape histograms, we determine the probability of phase trapping in the $\pm \varphi$-wells for different temperatures. Our experimental results qualitatively agree with theoretical predictions. In particular, we observe an onset of the butterfly effect with an oscillating probability of trapping. Unexpectedly, these probability saturates at a value different than $50 \%$ at low temparatures.
\end{abstract}

PACS numbers: $74.50 .+\mathrm{r}$, 85.25.Cp 05.60.Cd

Keywords: $\varphi$ Josephson junction, retrapping, escape

\section{INTRODUCTION}

The butterfly effect occurs in deterministic nonlinear systems and, in essence, means extreme sensitivity of the final state of the system to initial conditions ${ }^{1,2}$. The effect puts a clear distinction between determinism and predictability. A canonical example ${ }^{3}$ is the weather, which cannot be predicted reliably for more than 3-5 days in advance, although the computing power allows to make simulations much further forward. Deterministic chaotic systems must exhibit the butterfly effect. However, deterministic systems with dimensionality less than 3 cannot exhibit chaos, but nevertheless can exhibit the butterfly effect. Below we investigate experimentally one such system based on a Josephson $\varphi$ junction.

Josephson $\varphi$ junctions ( $\varphi$ JJs) attracted a lot of interest in the last few years, both theoretically ${ }^{4-14}$ and experimentally ${ }^{15-19}$, due to the peculiar physics and their properties. In general, a JJ can be imagined as a system where a particle with coordinate $\psi$ (Josephson phase) moves along a $1 \mathrm{D}$ potential $U_{0}(\psi)$. In the $\varphi$ JJ the potential $U_{0}(\psi)$ has the form of a $2 \pi$ periodic double well profile with degenerate minima at $\psi= \pm \varphi+2 \pi n$, where $0<\varphi<\pi$ depends on the parameters and $n$ is an integer.

In the ground state (no current applied) the phase is trapped in one of two wells of $U_{0}(\psi)$. Upon application of the bias current $I$ the potential will be tilted as $U(\psi)=U_{0}(\psi)-\psi I \Phi_{0} /(2 \pi)$, where $\Phi_{0} \approx 2.067 \mathrm{fWb}$ is the magnetic flux quantum. At some critical value of the bias current (tilt) the phase escapes from the corresponding well and starts sliding down the potential. Therefore, in experiment one can observe two critical currents $I_{c-}$ and $I_{c+}$, each corresponding to escape of the phase from different wells ${ }^{9,18}$. In general, $I_{c+} \neq I_{c-}$. Thus, the measurement of the critical current $\left(I_{c-}\right.$ or $\left.I_{c+}\right)$ can be used as a simple way to readout an unknown state $(-\varphi$ or $+\varphi)$ of the $\varphi \mathrm{JJ}^{18,19}$.

It is also interesting to understand in which of the two wells the phase is retrapped when the bias current (tilt) is decreased. In previous experiment ${ }^{18}$ we have noticed that the retrapping process depends strongly on temperature: for $T \gtrsim 2.4 \mathrm{~K}$ the destination well (state) is always $+\varphi$ (if the JJ returns to zero-voltage state from positive currents and voltages), while for low temperatures $T \sim 300 \mathrm{mK}$, the destination well is random. Theoretical analysis ${ }^{13}$ of a simplified deterministic model shows that the destination well is indeed $+\varphi$ at large damping $\alpha$. However it changes between $+\varphi$ and $-\varphi$ back and forth as $\alpha$ decreases. Actually, the intervals of $\alpha$ corresponding to the trapping in a particular well get shorter and shorter as $\alpha \rightarrow 0$. This, in fact, results in a butterfly effect in the limit $\alpha \rightarrow 0$, i.e., any tiny change (fluctuation) of the bias current or the damping will change the destination well. In the presence of noise (electronic or thermal), we expect ${ }^{13}$ that the probability $P(\alpha)$ to be trapped in a particular well should exhibit smeared oscillations and should saturate at 0.5 for $\alpha \rightarrow 0$.

In this tetterwork we present measurements of the probabilities of phase retrapping in the $\pm \varphi$ wells as a function of temperature and compare them with theoretical predictions. We note that the $\operatorname{model}^{13}$ represents a simplified version of the real system, resulting from several approximations: (a) an effective (spatially averaged) model was used, which works well only for very short JJs and reduces an infinite dimensional system to a $2 \mathrm{D}$ one (without chaos); (b) only "slow" (in comparison with the 


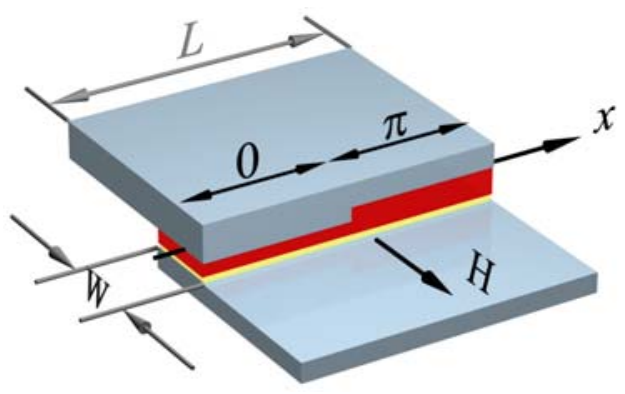

FIG. 1. Sketch of the sample under investigation - a (from bottom to top) $\mathrm{Nb}|\mathrm{AlO}| \mathrm{CuNi} \mid \mathrm{Nb}$ JJ of length $L \sim \lambda_{J}$ and width $w \ll L$. The critical temperature of the device $T_{c} \sim$ $9 \mathrm{~K}$. The magnetic field $H$ is applied along $y$ direction, see the figure.

retrappimg time) noise was treated ${ }^{13}$; (c) a linear damping was assumed ${ }^{13}$. Therefore, it is neccesary to check the predictions of the model ${ }^{13}$ experimentally.

Our results demonstrate a crossover from deterministic behavior of the probability to be trapped in the $-\varphi$ well $P_{-}(T)=0$ at high temperature $T>T^{*}$ to an oscillating probability $P_{-}(T)$ at the onset of the butterfly effect at $T<T^{*}$. However, at even lower temperatures $P_{-}(T)$ saturates at about 0.33 instead of 0.5 predicted theoretically. Possible reasons of this are discussed.

\section{EXPERIMENTAL RESULTS}

For our investigations we have used a superconductorinsulator-ferromagnet-superconductor (SIFS) JJ with a step-wise thickness of the F-layer, see Fig. 1, i.e., a JJ with one half bahaving like a conventional 0 JJ and the other half behaving like a $\pi$ JJ (further called $0-\pi$ JJ). This JJ is short in $x$ direction (the length $L$ is smaller than the Josephson length $\lambda_{J}$ ) and even smaller in $y$ direction $(w<L)$. Treating this JJ as a point-like device with the properties averaged along its length $L$, we obtain a $\varphi$ JJ with an effective (averaged) Josephson energy profile $U_{0}(\psi)$ looking like a $2 \pi$ periodic double well. Here $\psi$ is the average phase across the device. The sample described here was used in our previous works before ${ }^{18,19}$.

The current-voltage characteristic (IVC) at $H=0$ and the critical current dependence on the applied magnetic field $I_{c}(H)$ at $T=0.30 \mathrm{~K}$ are shown in Fig. 2(a,b). Here one can observe the presence of the two critical currents $I_{c-}$ and $I_{c+}$ in each direction of the bias ${ }^{20}$.

In our experiment we measured the probability to trap the phase in one of the two wells for different values of the temperature in the range $0.27 \mathrm{~K}<T<2.30 \mathrm{~K}$. Note that the damping $\alpha$ is a function of the temperature $T$ in our tunnel-like $\varphi$ JJ. So we change $T$ to change $\alpha$. We sweep the bias current $N=10^{4}$ times with a constant rate $\dot{I}=0.1194 \mathrm{~A} / \mathrm{s}$ at $H=0$ and obtain a histogram as the one shown in Fig. 2(c). In general, it consists of two peaks: one situated just below the fluctuation-free $I_{c-}$
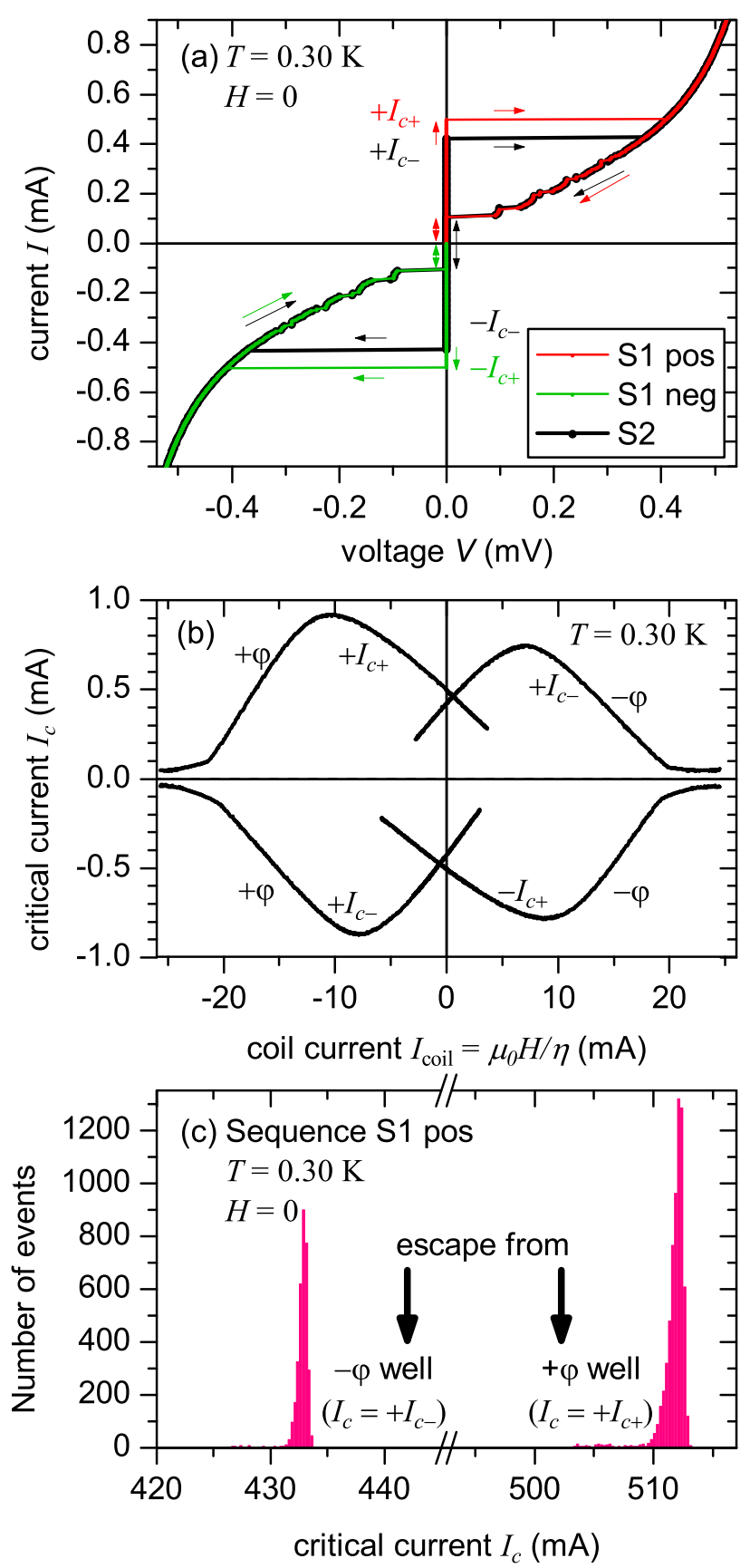

FIG. 2. (Color online) Current-voltage characteristic (a), critical current dependence on the magnetic field (b) and escape histogram (c) at $T=0.30 \mathrm{~K}$. In (a), different colors correspond to different sweep sequences of the bias current $I$. In (b), the magnetic field is applied in-plane of the junction by means of a coil with $\mu_{0} H=\eta \cdot I_{\text {coil }}$ with the coil factor $\eta \sim 5 \mu \mathrm{T} / \mathrm{mA}$. The histogram in (c), measured by using sequence $\mathrm{S} 1$ pos, shows two peaks corresponding to the two critical currents $I_{c \pm}$.

and another one below $I_{c+}$. The probability $P_{ \pm}=N_{ \pm} / N$ that the phase was trapped and then escapes from the $\pm \varphi$ well is proportional to the number of events $N_{ \pm}$in 
the corresponding peak in the histogram.

We performed the escape measurements using two different sweep sequences, refer to Fig. 2(a).

$\mathrm{S} 1$. In the sequence "S1 pos(neg)" we sweep the current $I$ from zero (zero voltage) to a maximum positive (negative) value with $V>0(V<0)$ at the McCumber branch. During this forward sweep we readout the value of the critical current and add this to the statistics to produce a histogram later on. Then we sweep back to $I=0$. During this sweep the phase is retrapped in one of the wells, $-\varphi$ or $+\varphi$ when the voltage jumps back to $V=0$. The value of the phase $(-\varphi$ or $+\varphi)$ will be read out during the next cycle.

$\mathrm{S} 2$. In the sequence "S2 pos(neg)" the sweep starts from the negative (positive) voltage state at the McCumber branch. Then the current $I$ is swept to a positive (negative) value up to the resistive branch. During this sweep the phase is retrapped when the current approaches $I=0$, but is still negative (positive), and then the critical current is readout (and added to statistics) when the voltage jumps from $V=0$ to $V>0(V<0)$. Finally, we sweep $I$ back to the initial value.

Note that the probabilities $P_{ \pm}$to be trapped in the $\pm \varphi$ wells (populations of histogram peaks) obtained using S1 and S2 are expected to be inverse, provided the potential $U_{0}(\psi)$ is symmetric, i.e., $P_{ \pm}^{\mathrm{S} 1}=P_{\mp}^{\mathrm{S} 2}$, because the trapping during $\mathrm{S} 1$ and $\mathrm{S} 2$ takes place at opposite values of the bias current (tilt). Also, the results of "pos" and "neg" sequences are opposite. Obviously, for any sequence $P_{-}+P_{+}=1$. Therefore, below, we discuss only $P_{-}(T)$ for "S1 pos" and the other $P(T)$ that are supposed to be the same $\left(P_{+}^{\mathrm{S} 1 \mathrm{neg}}, P_{+}^{\mathrm{S} 2 \mathrm{pos}}\right.$ and $\left.P_{-}^{\mathrm{S} 2 \text { neg }}\right)$ may be plotted in addition (see Fig. 3 below).

We have already seen $^{18}$ that for high damping the retrapping process is deterministic and we can predict the destination well of the Josephson phase. Namely, at $T>2.3 \mathrm{~K}$ the phase is always trapped in the $+\varphi$ well, i.e., $P_{-}=0$. However as the damping decreases, the destination well cannot be predicted and it depends on $\alpha$ and noise (thermal or electronic) ${ }^{13}$. In our experiment the damping should depend on temperature quite strongly as in any tunnel junction. Therefore we study $P_{-}(T)$ instead of the $P_{-}(\alpha)$ in experiment. Also, the noise is most probably dominated by electronic noise in the setup rather than by the thermal fluctuations in the JJ itself. Thus, it does not strongly depend on $T$.

In Fig. 3 we present the experimentally determined probability $P_{-}(T)$. The vertical line in the figure indicates the temperature $T^{*} \approx 2.25 \mathrm{~K}$ where the boundary between deterministic and non-deterministic retrapping is situated. For $T<T^{*}$ we observe an increase and several oscillations of the probability $P_{-}(T)$, qualitatively similar to the theoretical prediction ${ }^{13}$. Three peaks at $T=2.15 \mathrm{~K}, T=1.76 \mathrm{~K}$ and $T=1.56 \mathrm{~K}$ indicate an enhanced probability to be trapped in an "unnatural" $-\varphi$-well. Those peaks are the smeared traces of the regions where the phase is trapped in the $-\varphi$-well in the noiseless case. For $T<1.3 \mathrm{~K}$ the $P_{-}(T)$ saturates similar

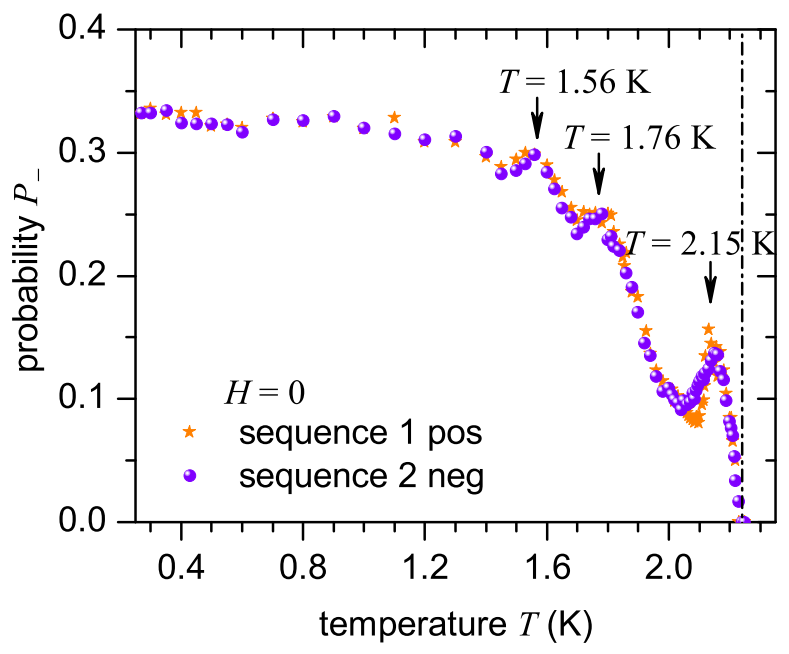

FIG. 3. (Color online) Retrapping probability $P_{-}$of the phase in the $-\varphi$ of the Josephson potential $U(\psi)$ for different temperatures $T$ at zero applied magnetic field $H$. The two set of data correspond to two different sweep sequence of the bias current $I$.

to the theoretical prediction ${ }^{13}$. However, the asymptotic value of $P_{-}$in this region is $\approx 0.33$ rather than 0.5 as expected from the theory.

Retrapping measurements with the other possible sweep sequences show similar results ("S2 neg" is shown in Fig. 3, "S1 neg" and "S2 pos" are not shown). The results also do not depend strongly on the ramp rate $\dot{I}$. Very similar results can be obtained by just moving the bias point along IVC with an unknown ramp rate defined by measurement electronics.

\section{DISCUSSION}

Here we discuss several possible reasons that can lead to the saturation value of $P_{-}(T)$ different from 0.5 .

a. Asymmetric $U_{0}(\psi)$. First, one of the possible reasons can be an asymmetry of the potential $U_{0}(\psi)$. The limit $\alpha \rightarrow 0$ implies that the retrapping current $I_{R} \rightarrow 0$. Therefore, in this limit, the potential is untilted and, in the case of a symmetric potential, one expects equal probabilities of trapping for both wells. If, however, the potential is asymmetric, $P_{-}(\alpha \rightarrow 0) \neq 0.5$.

Of course, the potential $U_{0}(\psi)$ in our $\varphi$ can be somewhat asymmetric, in particular because of nonuniformities along the JJ or because of a remnant magnetic field. However, an asymmetric potential will result in asymmetric critical currents $\pm I_{c+}$ and $\pm I_{c-}$. In our experiment, we can affect the asymmetry of the potential by applying an external magnetic field $H$. We have repeated the measurements shown in Fig. 3 applying a small ( $\left|I_{\text {coil }}\right|<1 \mathrm{~mA}$, see Fig. 2(b)) magnetic field such that the measurements always take place at symmetric critical currents. We have found that such a technique 
changes the $P_{-}(T)$ curve in the vicinity of the peaks, however, the saturation value remains almost unchanged.

One can further argue that the symmetry of the critical currents does not guarantee the symmetry of the potential, as the measurements of the critical currents are the measurements of the maximum slopes of the potential rather then the whole potential. Although, such a coinsidence is very unprobable. Nevertheless, we have generalized the deterministic model ${ }^{13}$ to the case of an asymmetric potential and weak noise (quasi-deterministic limit), i.e., the noise energy is smaller than the depth of each potential well, measured relative to the potential barrier separating the wells. Qualitatively one can say that at $\alpha \rightarrow 0$, the particle experiences several dephasings during the relaxation process and arrives to the decision point (last pass over the barrier separating the well) with a small, but random energy (velocity). Then one can conjecture that $P_{ \pm} \propto Q_{ \pm}$- the heat dissipated by the particle starting with zero velocity at the barrier during the "left and back" (along $-\varphi$ well) or "right and back" (along $+\varphi$ well) semiperiods. That is, the larger is the energy lost during traveling along a particular well, the larger is the probability to be trapped in this well. In the perturbation theory $\operatorname{limit}^{13}(\alpha \rightarrow 0)$

$$
Q_{ \pm}= \pm 2 \alpha \int_{\psi_{0}}^{ \pm \psi\left(U_{\mathrm{bar}}\right)} \sqrt{2\left[U_{\mathrm{bar}}-U_{0}(\psi)\right]} d \psi
$$

where $\psi_{0}$ is the phase corresponding to the "small" maximum of the potential $U_{0}(\psi)$ between the wells, i.e., the barrier, $U_{\mathrm{bar}}=U_{0}\left(\psi_{0}\right)$. The phases $\pm \psi\left(U_{\mathrm{bar}}\right)$ are the phases at the left and right slopes of the double-well potential were $U_{0}(\psi)=U_{\text {bar }}$. The final normalized expression for the probabilities is

$$
P_{ \pm}=\frac{Q_{ \pm}}{Q_{+}+Q_{-}}
$$

which is independent on $\alpha$.

To check our conjecture, we have performed direct numerical simulations of a particle falling into a double well potential subject to a weak noise, which confirms the prediction given by Eq. (2), see supplement.

Then, we have tried different asymmetric potentials, having the same symmetric $\pm I_{c+}$ and $\pm I_{c-}$ critical currents and estimated $P_{-}$using Eq. (2). It turns out that one can find some asymmetric profiles $U_{0}(\psi)$ that give $P_{-} \approx 0.33$, see supplement. However, for such profiles the dependence of $\pm I_{c \pm}(H)$ is not point symmetric like in experiment. Of course, one can further argue, that the magnetic field $H$ also has some asymmetric effect on $U_{0}(\psi)$, rather than just adding an odd function. The reason of this can be the same as the reason of asymmetry of $U_{0}(\psi)$ without magnetic field. This special asymmetry can make $\pm I_{c \pm}(H)$ symmetric as in experiment. However, again, such a coincidence is highly unlikely. Thus, to explain $P_{-} \approx 0.33$ with the help of an asymmetric potential, we have to make two improbable assumptions.
Finally, the strongest argument in favor of a symmetric potential is the fact that the dependences $P_{-}(T)$ obtained using S1 and $P_{+}(T)$ obtained using S2 almost coincide, see Fig. 3.

b. Saturation of damping $\alpha(T)$. Actually, the most simple and most probable reason of our observation is the saturation of damping $\alpha(T)$ at low temperatures caused, e.g., by leakage currents in the barrier. If the damping $\alpha(T)$ does not decrease further with $T \rightarrow 0$, but saturates below $T_{\text {sat }}$ at the value $\alpha_{\text {sat }}$, then in the $P_{-}(\alpha)$ plot we are able to go only down to $\alpha_{\text {sat }}$ where $P_{-}(\alpha)$ is not saturated yet but still performs decaying oscillations. So, what we see in the limit $T \rightarrow 0$ is then just $P_{-}\left(\alpha_{\text {sat }}\right)$, which happens to be 0.33 in our system.

To check this, we determine $\alpha(T)$ from IVCs taken at different temperatures. The pitfall here is that our SIFS JJ is not RSJ-like, i.e., the resistance is voltagedependent and, strictly speaking, $\alpha$ is not defined. However, our task is not to determine the exact value of $\alpha$, but rather to see whether it saturates or not, and at which $T$. For this purpose we have performed a fitting of the low-voltage part of experimental IVCs by solving the $1 \mathrm{D}$ perturbed sine-Gordon equation for a $0-\pi \mathrm{JJ}$ with constant ( $x$ independent) damping $\alpha$, see Fig. 1. Before doing these fits, we have estimated the other key parameters of our JJ such as its length Josephson $\lambda_{J}$, and critical current densities $j_{c 0}$ and $j_{c \pi}$ of the 0 and the $\pi$ parts, respectively, by fitting the numerically obtained $I_{c}(H)$ dependence to the experimentally measured one. The dependence $\alpha(T)$ is then obtained from fitting IVCs at different temperatures and is shown in Fig. 4. One sees that at $T<T_{\text {sat }} \approx 1.2 \mathrm{~K}$ the damping $\alpha(T)$ saturates, presumably due to leakage. Self-heating at $T \sim 1 \mathrm{~K}$ is still a minor effect.

Knowing the $\alpha(T)$ dependence we have also performed simulations of the retrapping probability for different values of $T$. Since our $0-\pi$ JJ is not extremely short, the model of a point-like JJ with bi-harmonic averaged current-phase relation is valid only qualitatively. Therefore, for simulations we again used a $0-\pi$ JJ of finite length to be as close as possible to experiment. The thermal noise term was taken as $\propto T$. The results show a behavior of $P_{-}(T)$ qualitatively similar to the experimental one in Fig. 3, i.e., $P_{-}(T)$ makes a few oscillations and saturates at a value different from 0.5 (e.g., 0.21) as $T$ decreases (not shown). The discrepancy between the experimentally and numerically obtained saturation value of $P_{-}(T \rightarrow 0)$ can be caused by some fine details such as non-linearity of the damping in experiment or $x$ dependent damping in the sample (different damping in 0 and $\pi$ parts).

Nevertheless we have checked that in our simulations $P_{-} \rightarrow 0.5$ when the damping becomes much smaller than $\alpha_{\text {sat }}=0.057$ - the saturation value in Fig. 4. During this simulation the noise was kept constant and corresponding to a value $T=300 \mathrm{mK}$ (constant noise amplitude independent on $\alpha$ ). These results support our claim that the saturation of the damping $\alpha(T)$ leads to 
$P_{-}(T \rightarrow 0) \neq 0.5$.

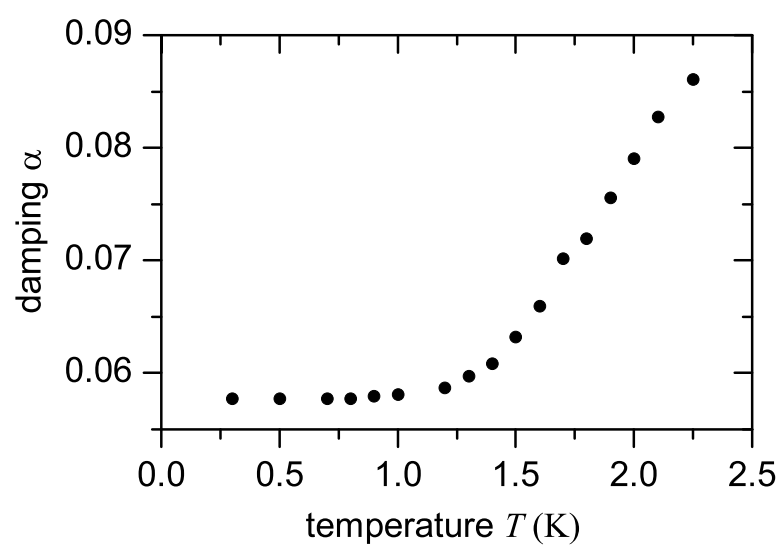

FIG. 4. Plot of $\alpha(T)$ obtained by means of fitting IVCs as described in the text.

Finally, we would like to estimate whether the peak width in the $P_{-}(T)$ plot in Fig. 3 is in agreement with the noise level. The noise amplitude in our setup $\sqrt{\left\langle\delta I^{2}\right\rangle} \sim$ $1 \mu \mathrm{A}$. In normalized units $\sqrt{\left\langle\delta \gamma^{2}\right\rangle} \sim 0.002$. This translates into the "noise" in $\alpha$ as $\sqrt{\left\langle\delta \alpha^{2}\right\rangle}=\sqrt{\left\langle\delta \gamma^{2}\right\rangle} / I\left(\Gamma_{0}\right)$, where ${ }^{13} I\left(\Gamma_{0}\right) \sim 1$. Using the $\alpha(T)$ plot in Fig. 4 , one can measure that above $1.5 \mathrm{~K}$ the slope $\partial \alpha / \partial T \approx 1 /(60 \mathrm{~K})$. This gives

$$
\sqrt{\left\langle\delta T^{2}\right\rangle} \sim \sqrt{\left\langle\delta \alpha^{2}\right\rangle} \frac{\partial T}{\partial \alpha} \sim 0.002 \cdot 60 \mathrm{~K}=0.12 \mathrm{~K},
$$

in good agreement with Fig. 3. Note, however, that at $T<1 \mathrm{~K}$, where the $\alpha(T)$ dependence saturates, the slope $\partial \alpha / \partial T$ vanishes, which leads to $\sqrt{\left\langle\delta T^{2}\right\rangle} \rightarrow \infty$. In this sense, a weakly pronounced maximum at $T \sim 1 \mathrm{~K}$ can be yet another stretched oscillation.

\section{CONCLUSIONS}

We have performed measurements of the phase escape and retrapping from/in two distinct states $\pm \varphi$ of a Josephson $\varphi$ junction in a temperature range from $0.3-3 \mathrm{~K}$. We have seen that retrapping is deterministic above some damping (temperature) $\alpha^{*}\left(T^{*}\right)$. At $\alpha<\alpha^{*}$ $\left(T<T^{*}\right)$ the probability of trapping in the unnatural well $P_{-}(\alpha(T))$ grows and oscillates demonstrating the onset of the butterfly effect as predicted earlier ${ }^{13}$. However, we observe that the probability $P_{-}(T)$ saturates at a level different from 0.5 most probably because $\alpha(T)$ saturates at its minimum value $\alpha_{\text {sat }}$ for $T<T_{\text {sat }}$. Thus, we were not able to penetrate deep into the region of the butterfly effect. Further experiments with the $\varphi$ JJs showing lower damping, e.g., effective $\varphi$ JJ based on dc SQUID $^{21}$, may help to move further in this direction.

\section{ACKNOWLEDGMENTS}

R. M. gratefully acknowledges support by the Carl Zeiss Stiftung. This work was supported by the Deutsche Forschungsgemeinschaft (DFG) via project No. GO$1106 / 5$, via project A5 within SFB/TRR-21, and by the EU-FP6-COST action MP1201. 
* Current address: Physikalisches Institut, Karlsruher Institut für Technologie, 76131 Karlsruhe, Germany

1 A. Scott, Encyclopedia of Nonlinear Science (Taylor \& Francis Group, 2005).

2 J. Sprott, Chaos and Time-Series Analysis (Oxford University Press, 2003).

${ }^{3}$ E. N. Lorenz, J. Atmos. Sci. 20, 130 (1963).

${ }^{4}$ R. G. Mints, Phys. Rev. B 57, R3221 (1998).

5 A. Buzdin and A. E. Koshelev, Phys. Rev. B 67, 220504(R) (2003), arXiv:cond-mat/0305142.

6 Y. Tanaka and S. Kashiwaya, Phys. Rev. B 56, 892 (1997).

7 A. Gumann and N. Schopohl, Phys. Rev. B 79, 144505 (2009).

8 N. G. Pugach, E. Goldobin, R. Kleiner, and D. Koelle, Phys. Rev. B 81, 104513 (2010).

9 E. Goldobin, D. Koelle, R. Kleiner, and A. Buzdin, Phys. Rev. B 76, 224523 (2007), arXiv:0708.2624.

10 E. Goldobin, D. Koelle, R. Kleiner, and R. G. Mints, Phys. Rev. Lett. 107, 227001 (2011), arXiv:1110.2326.

11 S. V. Bakurskiy, N. V. Klenov, T. Y. Karminskaya, M. Y. Kupriyanov, and A. A. Golubov, Supercond. Sci. Technol. 26, 015005 (2013).

12 D. M. Heim, N. G. Pugach, M. Y. Kupriyanov, E. Goldobin, D. Koelle, and R. Kleiner, J. Phys. Cond. Mat. 25, 215701 (2013), arXiv:1302.4398 [cond-mat.suprcon].

13 E. Goldobin, R. Kleiner, D. Koelle, and R. G. Mints, Phys. Rev. Lett. 111, 057004 (2013), arXiv:1307.8042 [cond- mat.supr-con].

14 A. Lipman, R. G. Mints, R. Kleiner, D. Koelle, and E. Goldobin, Phys. Rev. B 90, 184502 (2014), arXiv:1208.4057.

15 E. Il'ichev, M. Grajcar, R. Hlubina, R. P. J. IJsselsteijn, H. E. Hoenig, H.-G. Meyer, A. Golubov, M. H. S. Amin, A. M. Zagoskin, A. N. Omelyanchouk, and M. Y. Kupriyanov, Phys. Rev. Lett. 86, 5369 (2001).

16 G. Testa, E. Sarnelli, A. Monaco, E. Esposito, M. Ejrnaes, D.-J. Kang, S. H. Mennema, E. J. Tarte, and M. G. Blamire, Phys. Rev. B 71, 134520 (2005).

17 R. G. Mints, I. Papiashvili, J. R. Kirtley, H. Hilgenkamp, G. Hammerl, and J. Mannhart, Phys. Rev. Lett. 89, 067004 (2002).

${ }^{18}$ H. Sickinger, A. Lipman, M. Weides, R. G. Mints, H. Kohlstedt, D. Koelle, R. Kleiner, and E. Goldobin, Phys. Rev. Lett. 109, 107002 (2012), arXiv:1207.3013 [cond-mat.supr-con].

19 E. Goldobin, H. Sickinger, M. Weides, N. Ruppelt, H. Kohlstedt, R. Kleiner, and D. Koelle, Appl. Phys. Lett. 102, 242602 (2013).

20 Another main feature of the $\varphi \mathrm{JJ}$ is visible in the $I_{c}(H)$ curve (Fig. 2(b)), which has cusp-like minima, that are point symmetrically shifted from $H=0$.

21 E. Goldobin, D. Koelle, and R. Kleiner, Phys. Rev. B 91, 214511 (2015). 